\title{
Matlab implementation of ECG signal processing
}

\author{
V.Viknesh \& P. Ram Prashanth \\ Final year ECE Panimalar Institute Of Technology Chennai.
}

\begin{abstract}
Signal processing today is performed in vast majority of systems for ECG analysis and interpretation. The objective of ECG signal processing is manifold and comprises the improvement of measurement accuracy and reproducibility and the extraction of information not readily available from the signal through visual assessment. In many situations, the ECG is recorded during ambulatory or strenuous conditions such that the signal is corrupted by different types of noise, sometimes originating from other physiological process of the body. Hence noise reduction represents another important objective of ECG signal processing. The paper mainly focuses on implementing the present day trends and procedures in the processing of ECG signals using software (MATLAB). The implementation process helps us to understand the drawbacks and difficulties of such methods and gives us an opportunity to work out towards finding a better solution. Such a solution would satisfy the scope of improvement expected in the technologies, used at present.
\end{abstract}

Keywords: $E C G$, baseline wander, powerline interference, $Q R S$ detection

\section{INTRODUCTION}

Electrocardiographic signals may be recorded on a long timescale (i.e., several days) for the purpose of identifying intermittently occurring disturbances in the heart rhythm. As a result, the produced ECG recording amounts to huge data sizes that quickly fill up available storage space. Transmission of signals across public telephone networks is another application in which large amounts of data are involved. For both situations, data compression is an essential operation and, consequently, represents yet another objective of ECG signal processing. It is a basic set of algorithms that condition the signal with respect to different types of noise and artifacts, detect heartbeats, extract basic ECG measurements of wave amplitudes and durations, and compress the data for efficient storage or transmission; the block diagram in Fig. 1 presents this set of signal processing algorithms. Although these algorithms are frequently implemented to operate in sequential order, information on the occurrence time of a heartbeat, as produced by the QRS detector, is sometimes incorporated into the other algorithms to improve performance. The complexity of each algorithm varies from application to application.

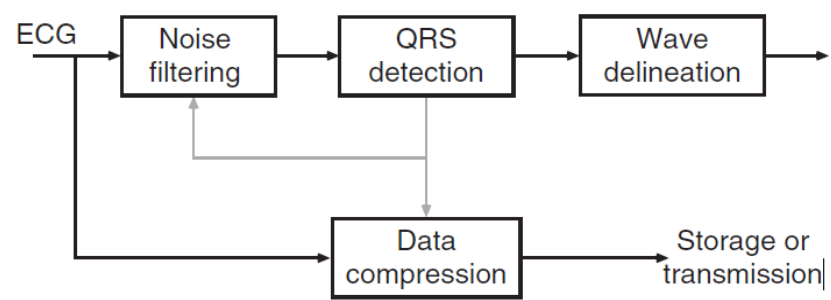

Fig. 1 The basic block diagram of ECG signal processing

\section{Methods To ObTain SOURCE OF ECG}

There are several methods to obtain the source of ECG.The one which can be compatible with matlab and the one that is capable of illustrating the steps of processing is chosen. Two such methods are discussed below.

\section{A. Use of inbuilt matlab generator}

The command that is to be used to obtain the source of ECG from matlab is: $>>\operatorname{ecg}(500)$ ECG(L) generates a piecewise linear ECG signal of length L. The output waveform obtained on execution of such an instruction is: 


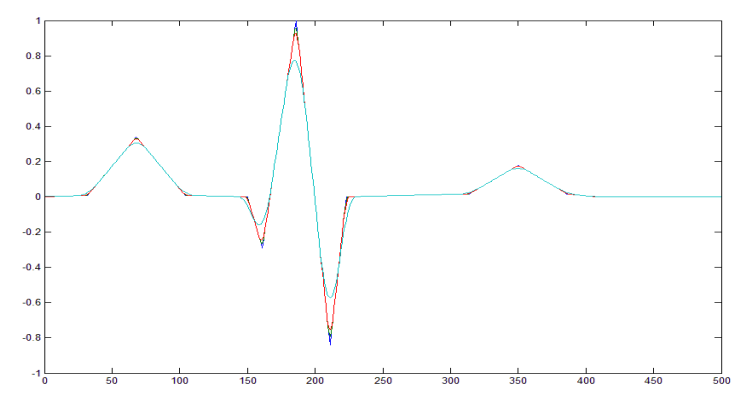

Fig. 2 waveform of ECG from matlab inbuilt generator

The signal obtained doesn't exhibit any noise or baseline wander hence the processing of such a signal is undesirable

\section{B. Use of ECG values from a database}

- The values from the database needs to be stored in the form of matlab compatible file

- Then the value is imported on to matlab workspace.

Example: Here the variable used for proceeding with further processing is "ecg ". The properties of the variable "ecg" is as follows:

\begin{tabular}{|l|l|l|l|}
\hline Name & Size & Bytes & class \\
\hline ecg & $4170 * 1$ & 33360 & Double array \\
\hline
\end{tabular}

The waveform given by these values are as follows: $>>$ plot(ecg)

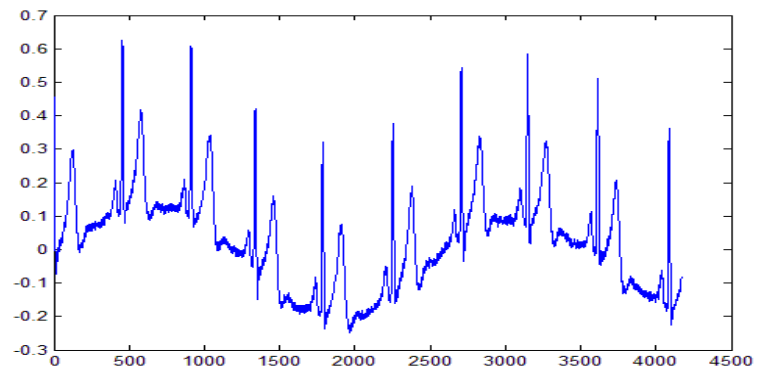

Fig. 3 waveform of ECG obtained from a database

The signal obtained exhibits noise and baseline wander hence the processing of such signal is explanatory at each stage

\section{ECG PRE-PROCESSING}

Considerable attention has been paid to the design of techniques such as filters for the purpose of removing baseline wander and power line interference; both types of disturbance imply the design of a narrowband filter. Removal of noise because of muscle activity represents another important filtering problem being much more difficult to handle because of the substantial spectral overlap between the ECG and muscle noise. Muscle noise present in the ECG can; however, be reduced whenever it is appropriate to employ techniques that benefit from the fact that the ECG is a recurrent signal. For example, ensemble averaging techniques can be successfully applied to time-aligned heartbeats for reduction of muscle noise. The filtering techniques are primarily used for pre-processing of the signal and have as such been implemented in a wide variety of systems for ECG analysis. It should be remembered that filtering of the ECG is contextual and should be performed only when the desired information remains undistorted. This important insight may be exemplified by filtering for the removal of power line interference. Such filtering is suitable in a system for the analysis of heart rate variability, whereas it is inappropriate in a system for the analysis of micro potentials; as such potentials spectrally overlap the power line interference. 


\section{A. Baseline wander}

Removal of baseline wander is required in order to minimize changes in beat morphology that do not have cardiac origin, which is especially important when subtle changes in the "lowfrequency" ST segment are analysed for the diagnosis of ischemia, which may be observed, for example, during the course of a stress test. The frequency content of baseline wander is usually in the range below $0.5 \mathrm{~Hz}$; however, increased movement of the body during the latter stages of a stress test further increases the frequency content of baseline wander.

Matlab coding to remove baseline wander:

$\%$ code for removal of baseline wander $\%$

for $\mathrm{i}=1: 1:$ length(ecg)

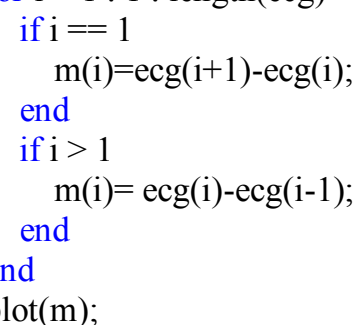

The waveform given by these values is as follows:

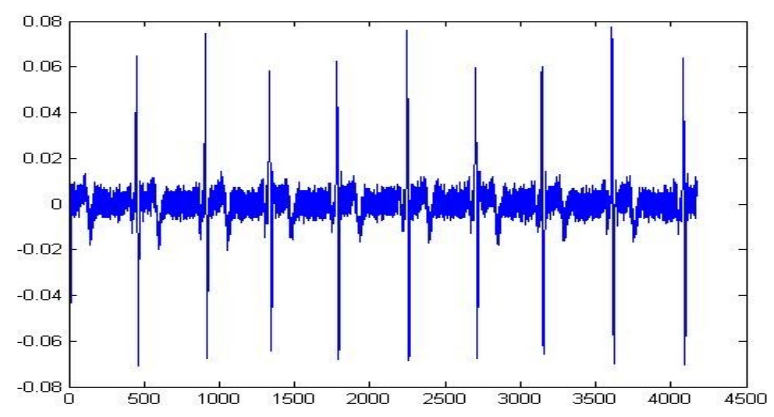

Fig. 4 waveform with baseline wander removed

\section{B. Powerline interference}

Electromagnetic fields caused by a powerline represent a common noise source in the ECG that is characterized by 50 or $60 \mathrm{~Hz}$ sinusoidal interference, possibly accompanied by a number of harmonics. Such narrowband noise renders the analysis and interpretation of the ECG more difficult, as the delineation of low-amplitude waveforms becomes unreliable and spurious waveforms may be introduced. Although various precautions can be taken to reduce the effect of powerline interference, for example, by selecting a recording location with few surrounding electrical devices or by appropriately shielding and grounding the location, it may still be necessary to perform signal processing to remove such interference. Several techniques have been presented for this purpose, ranging from straightforward linear, band stop filtering to more advanced techniques that handle variations in powerline frequency and suppress the influence of transients manifested by the occurrence of QRS complexes.A major concern when filtering out powerline interference is the degree to which the QRS complexes Influence the output of the filter. The QRS complex acts, in fact, as an unwanted, large-amplitude impulse input to the filter. As linear, time-invariant notch filters are generally more sensitive to the presence of such impulses, powerline filters with a nonlinear structure may be preferable. In order to assure that a filter does not introduce unacceptable distortion, its performance should be assessed by means of simulated signals so that distortion can be exactly quantified.

Matlab coding:

Procedure employing wavelet transformation:

1. Type 'wave menu' on the matlab main window

2. Now choose the 'wavelet packet 1 dimensional 'option

3. Then load the baseline wander removed noisy variable from the workspace

4. Now perform analysis by choosing 'analyse' option

5. Similarly denoise the signal by selecting 'denoise' option

6. Now save the denoised signal for further processing 
Execution:

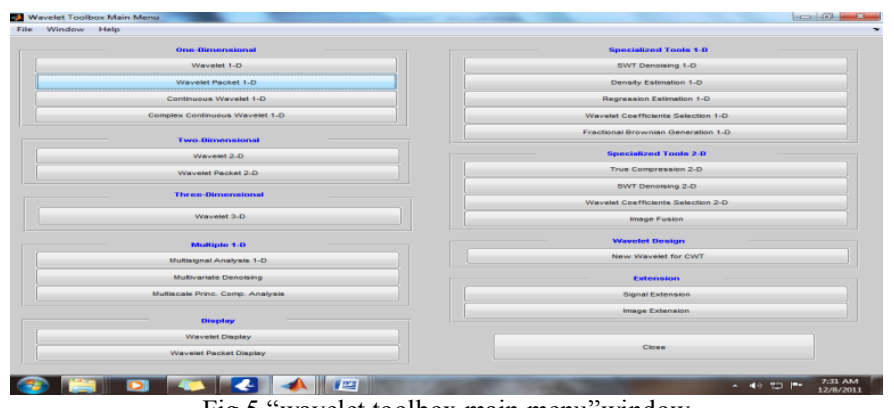

Fig.5 "wavelet toolbox main menu"window

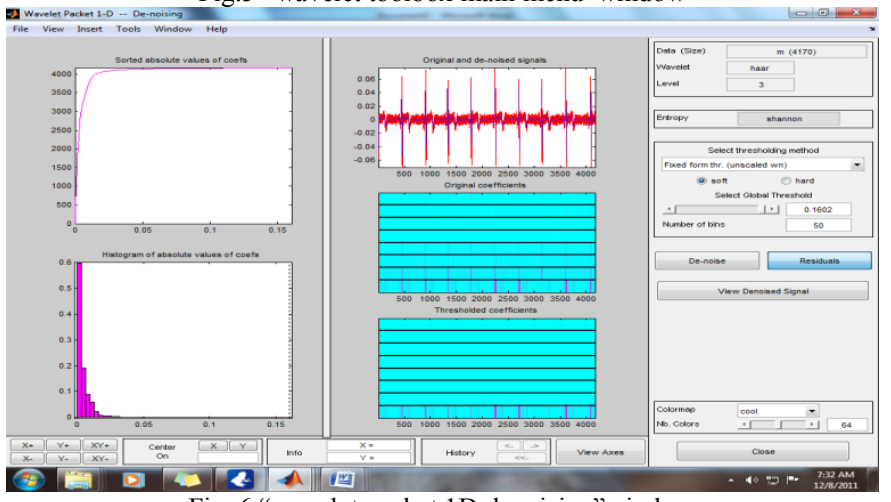

Fig. 6 "wavelet packet 1D denoising"window

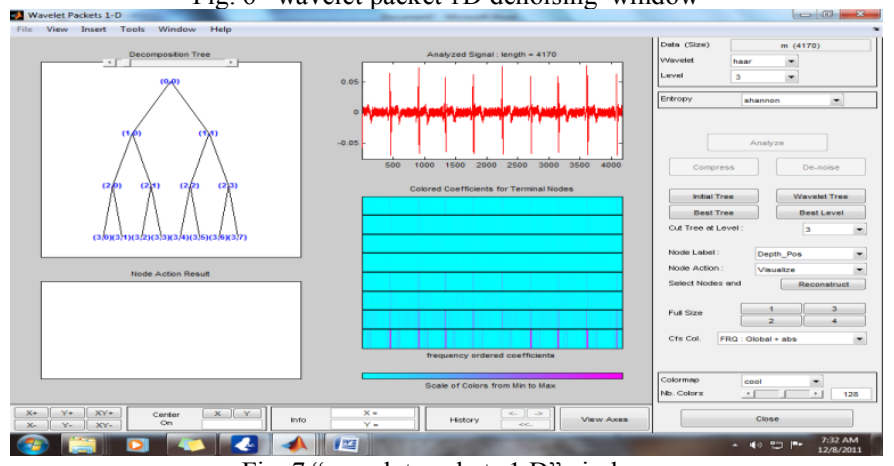

Fig. 7 "wavelet packets 1 D"window

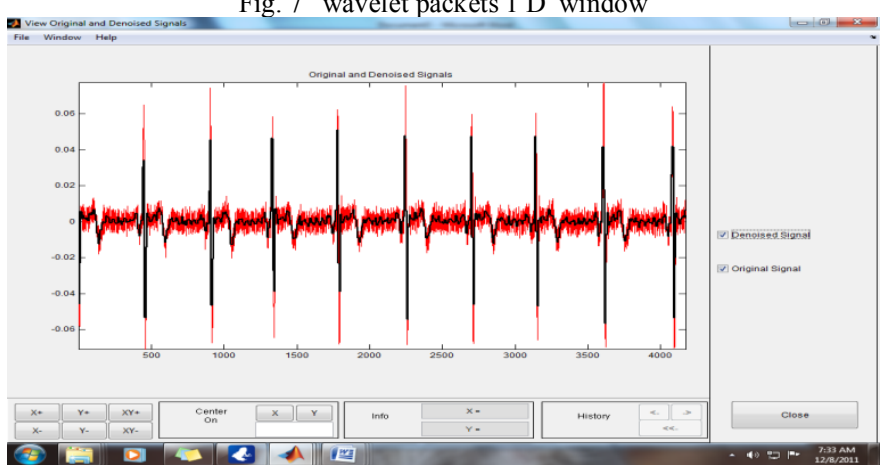

Fig. 8 "view original and denoised signal window"

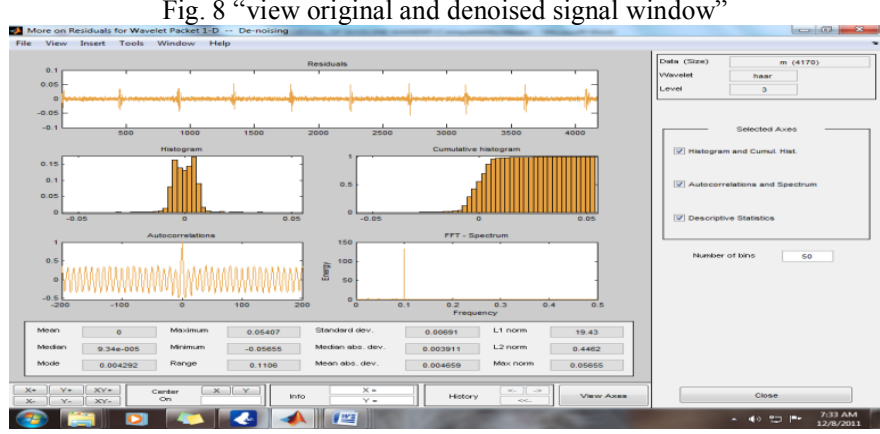

Fig. 9 "more on residuals of wavelet packet 1D denoising "window 
The waveform given by these values is as follows:

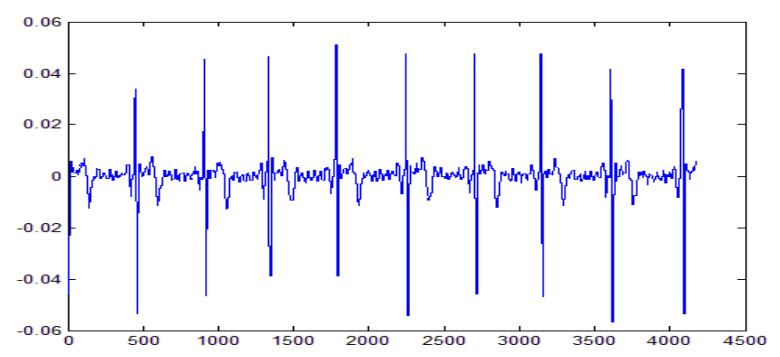

Fig. 10 waveform with powerline interference removed

\section{QRS DETECTION}

The presence of a heartbeat and its occurrence time is basic information required in all types of ECG signal processing. As the QRS complex is that waveform that is most easily discerned from the ECG, beat detection is synonymous to the detection of QRS complexes. The design of a QRS detector is of crucial importance because poor detection performance may propagate to subsequent processing steps and, consequently, limit the overall performance of the system. Beats that remain undetected constitute a more severe error than do false detections; the former type of error can be difficult to correct at a later stage in the chain of processing algorithms, whereas, hopefully, false detections can be eliminated by, for example, performing classification of QRS morphologies. A QRS detector must be able to detect a large number of different QRS morphologies in order to be clinically useful and able to follow sudden or gradual changes of the prevailing QRS morphology. Furthermore, the detector must not lock onto certain types of rhythm, but treat the next possible event as if it could occur at almost any time after the most recently detected beat. Several detector-critical types of noise and artifacts exist depending on the ECG application of interest. The noise may be highly transient in nature or be of a more persistent nature, as exemplified by the presence of powerline interference. In the case of an ECG recording with episodes containing excessive noise, it may be necessary to exclude such episodes from further analysis.

Matlab coding:

$\mathrm{a}=\max ($ denoisd);

past_pos $=1$;

count $=0$

for $\mathrm{i}=1: 1:$ length(denoisd)

if $\operatorname{denoisd}(\mathrm{i})<(0.60 * \mathrm{a})$

continue

else if (i-past_pos) $>(10)$

count $=$ count +1 ;

$\operatorname{pos}($ count $)=\mathrm{i}$;

$\operatorname{val}($ count $)=$ denoisd $(\mathrm{i})$;

past pos $=\mathrm{i}$;

end

end

end

stem(pos,val);

The graph obtained after the detection of QRS complexes is as follows:

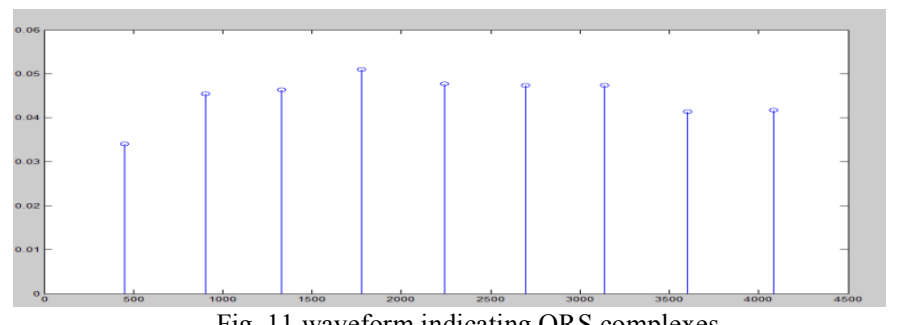

Fig. 11 waveform indicating QRS complexes 


\section{DATA COMPREsSion}

The overall goal is to represent a signal as accurately as possible using the fewest number of bits, by applying either lossless compression, in which the reconstructed signal is an exact replica of the original signal, or lossy compression, in which the reconstructed signal is allowed to differ from the original signal. With lossy compression, a certain amount of distortion has to be accepted in the reconstructed signal, although the distortion must remain small enough not to modify the diagnostic content of the ECG. For both types of compression, it may be necessary to perform noise filtering of the ECG signal before it is subjected to data compression.

As a wide range of clinical examinations involve the recording of ECG signals, huge amounts of data are produced not only for immediate scrutiny, but also for storage in a database for future retrieval and review. It is well-known that the availability of one or several previous ECG recordings improves diagnostic accuracy of various cardiac disorders, including myocardial infarction. Today, such serial ECG comparison encompasses short-duration recordings acquired during rest, but may in the future encompass long signals, for example, acquired during stress testing or ambulatory monitoring. Although hard disk technology has undergone dramatic improvements in recent years, increased disk size is paralleled by the ever-increasing wish of physicians to store more information. In particular, the inclusion of additional ECG leads, the use of higher sampling rates and finer amplitude resolution, the inclusion of other, non cardiac signals such as blood pressure and respiration, and so on, lead to rapidly increasing demands on disk size. It is evident that efficient methods of data compression will be required for a long time to come.

An ECG signal exhibits a certain amount of redundancy, as manifested by correlation between adjacent samples, the recurrence of heartbeats with similar morphology, and the relative resemblance between different leads. Considerable savings can be achieved in terms of storage capacity and transmission time by exploiting the different types of redundancy so that each sample can be represented by fewer bits than in the original signal. Hence, the data compression algorithm should account for the fact that the signal contains recurrent heartbeats, often with similar morphology, and that the signal is, almost invariably, a multilead recording. Equally important, it must account for the fact that both small- and large-amplitude waveforms are present in the signal, carrying important diagnostic information, whereas the isoelectric line contains negligible information.

The outcome of data compression is critically dependent on the sampling rate and the number of bits used to represent each sample of the original signal.

Matlab coding:

$>>$ min_val=min $(1000 *$ denoisd $)$;

$>>$ approx_val=ceil( $1000 *$ denoisd);

$>>$ approx_str=approx_val-min_val;

$>$ bin_val=dec2bin((approx_str $))$;

$>$ dec_val=bin2dec(bin_val);

$>$ inter_val=dec_val+min_val;

$>$ final_res=(inter_val/1000);

$>$ plot(final_res);

$>$ hold all;

> plot(denoisd);

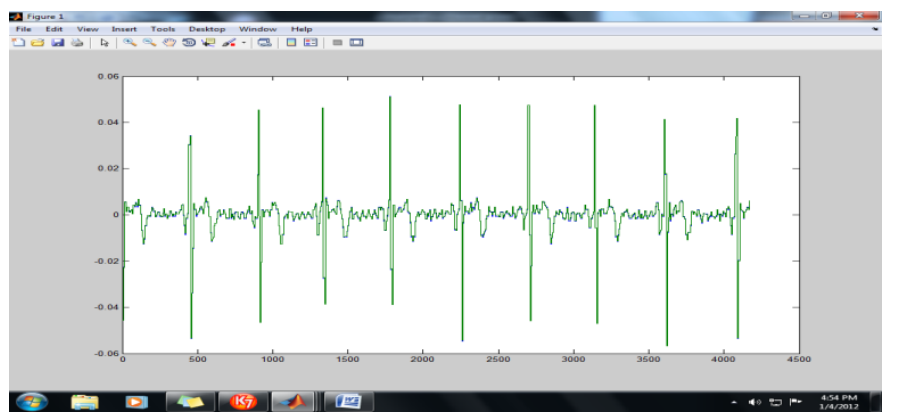

Fig. 12 waveform indicating the input and output of a data compression and expansion scheme with a scaling factor of 1000 


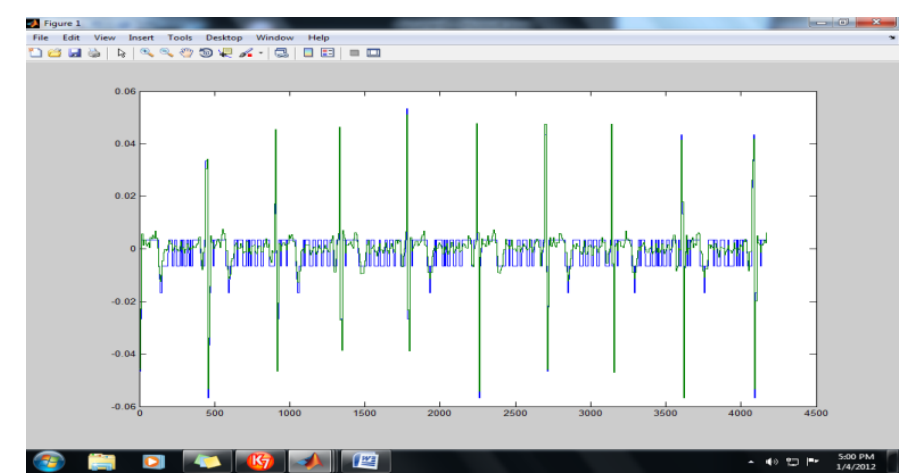

Fig. 13 waveform indicating the input and output of a data compression and expansion scheme with a scaling factor of 100

\section{PERFORMANCE EVALUATION}

The percentage root mean-square difference (PRD) is a frequently employed distortion measure that quantifies the error between the original signal and the reconstructed signal, defined by

$$
\mathscr{P}_{\mathrm{PRD}}=100 \cdot \sqrt{\sum_{n=0}^{N-1}(x(n)-\tilde{x}(n))^{2} / \sum_{n=0}^{N-1} x^{2}(n)},
$$

This is most commonly employed to estimate the distortion. However this method also imposes some drawbacks. That is during the evaluation of PRD for large amplitude signals, this measure always result in a low value

So we move on to a normalized parameter denoted by $\mathrm{P}_{\mathrm{rms}}$

$$
\mathscr{P}_{\mathrm{RMS}}=\sqrt{\frac{1}{N} \sum_{n=0}^{N-1}(x(n)-\tilde{x}(n))^{2}} .
$$

Matlab coding to evaluate the performance of data compression schemes used:

$\mathrm{N}=$ length(denoisd);

sum $=0$;

for $\mathrm{i}=1: 1: \mathrm{N}$

sum=sum+((denoisd(i)-final_res(i)).^2);

end

sum_sqr=((1/N)*(sum));

sum_sqr

rms_err=sqrt(sum_sqr);

rms_err

Output:

Case 1:

(Scaling factor used is 100)

sum_sqr $=$

$1.2655 \mathrm{e}-005$

rms_err $=0.0036$

Case 2:

(Scaling factor used is 1000)

sum_sqr $=$

$1.0094 \mathrm{e}-007$

rms err $=$

3. $\overline{17} 71 \mathrm{e}-004$

Thus the observations reveal the presence of greater noise and error in the usage of smaller scaling factor

\section{CONCLUSIONS}

Thus such an implementation serves as an initiative towards the development of a computerized diagnosis system. Further there are other provisions to convert the matlab programs to embedded c programs 
that tries to exhibit an increased performance over the hardware implementation of the same. To add these systematic approaches are in the way of their progress in the fields of present day medicine like biotelemetry etc.

\section{ACKNOWLEDGMENT}

I would like to express my gratitude to all those who had been a support during the completion of this paper. I would like to thank my college for proving a support and serving as a backbone during the paper completion. I would like to thank all my friends to have provided useful and constructive criticisms during the due course of completion.

\section{REFERENCES}

[1] Wiley Encyclopedia of Biomedical Engineering,2006 edition by John Wiley \& Sons, Inc.

[2] The official website of matlab - www.mathworks.com( for the matlab documentation) Design of ECG Signal Acquisition and Processing System (2012). Zeli Gao; Jie Wu; Jianli Zhou; Wei Jiang; Lihui Feng

[4] Implementation of ECG signal processing and analysis techniques in digital signal processor based system (2009). Balasubramaniam, D.; Nedumaran, D.

[5] New aspects in ECG signal processing using adaptive filters (2011). Tudosa, I.; Adochiei, N.I.; Ciobotariu, R.

[6] Processing ECG signals using rational function systems (2012).Locsi, L.; Kovacs, P.

[7] Evaluation of novel ECG Signal Processing on Quantificatiojn of Transient Ischemia amd Baseline wander suppression (2007). Kostic, M.N.; Fakhar, S.; Foxall, T.; Drakulic, B.S.; Krucoff, M.W.

[8] Image processing on ECG chart for ECG signal recovery (2009).Shen, T.W.; Laio, T.F.

[9] ECG signal acquisition and analysis for telemonitoring (2010).Plesnik, E.; Malgina, O.; Tasič, J.F.; Zajc, M.

[10] Power- line Interference Detection and Suppression in ECG Signal Processing (2008). Yue-Der Lin; Yu Hen Hu 